\title{
Evaluation of Risk Factors for Primary Fracture in Elderly Patients with Osteoporosis
}

\author{
Xiao Li ${ }^{1}$, Jun Wu ${ }^{1}$, Xiang Li ${ }^{1}$, Ka Li Frankie Leung ${ }^{1,2}$, Tak Man Wong ${ }^{1,2, ~ *, ~}$ \\ Xinshuo Christian Fang ${ }^{1,2}$ \\ ${ }^{1}$ Shenzhen Key Laboratory for Innovative Technology in Orthopaedic Trauma, Guangdong Engineering Technology Research Canter for \\ Orthopaedic Trauma Repair, Department of Orthopaedics and Traumatology, The University of Hong Kong-Shenzhen Hospital, Shenzhen, \\ China \\ ${ }^{2}$ Department of Orthopaedics and Traumatology, The University of Hong Kong, Queen Mary Hospital, Hong Kong
}

\author{
Email address: \\ wongtm@hku.hk (T. M. Wong) \\ ${ }^{*}$ Corresponding author
}

\section{To cite this article:}

Xiao Li, Jun Wu, Xiang Li, Ka Li Frankie Leung, Tak Man Wong, Xinshuo Christian Fang. Evaluation of Risk Factors for Primary Fracture in Elderly Patients with Osteoporosis. American Journal of Health Research. Vol. 9, No. 5, 2021, pp. 198-203. doi: 10.11648/j.ajhr.20210905.18

Received: August 17, 2021; Accepted: August 30, 2021; Published: September 15, 2021

\begin{abstract}
Introduction: Osteoporosis is one of the major fundamental causes of fractures in individuals over 50 years old. Preventing the first fragility fracture is the most cost-effective strategy for addressing osteoporosis. Therefore, identifying individuals with a high risk of developing osteoporotic fractures is important to save limited medical resources. The Fracture Risk Assessment Tool (FRAX) has been used globally for assessing fracture risk. However, the accuracy of FRAX still needs to be improved partially because of the differences in race and socioeconomic status among nationalities. Methods: In this study, we evaluated the effectiveness of FRAX in Chinese people. The factors not involved in FRAX were also evaluated for a correlation with osteoporotic fracture risks. Results: Age, smoking status, alcohol intake, family history of osteoporotic fracture, diabetes mellitus type II, Charlson Index, vitamin D intake, calcium intake, muscle strength, modified Barthel Index, the 3-level version of EuroQol five dimensions questionnaire, and bone mineral density demonstrated significant differences between the fracture and control groups. Our results also demonstrated that dual-energy X-ray absorptiometry (DEXA)-diagnosed osteoporosis (T $\leq$ -2.5) was the independent fracture risk factor. The effects of age, muscle strength, and Charlson Index on DEXA were found to be statistically significant. People old over 60, muscle strength test supine leg lift less than 20 times per minute, aCCI scores greater than or equal to 2 , had lower DEXA T values $(\mathrm{T} \leq-2.5)$. Discussion: This work was a single-center study, showed social economic status bias, and featured a limited number of cases. Therefore, multi-center studies are necessary in the future. Conclusions: This study revealed that FRAX should be improved further in combination with other risk factors, including aCCI, calcium intake, and muscle strength.
\end{abstract}

Keywords: Osteoporosis, Fracture, FRAX, Risk Factor

\section{Introduction}

Osteoporosis is a systemic metabolic skeletal disease characterized by decreasing bone mass and bone mineral density (BMD), as well as deteriorating microarchitecture in bone tissues, leading to bone fragility and elevated fracture risk $[1,2]$. Osteoporosis is one of the major fundamental causes of fractures in individuals aged over 50, with potentially serious and complex complications, including bedsores, renal failure, and pneumonia, and it is associated with increased relative mortality [3]. It is a disease largely associated with the aging population, as a number of mechanical, hormonal, behavioral, and other physiological changes that occur across the human lifespan [4]. Postmenopausal women are more susceptible to osteoporotic fracture [3, 5]. A recent study showed that in China, the prevalence of osteoporosis in the elderly over 50 years old was $19.2 \%$, with $6 \%$ in men and $32.1 \%$ in women. The prevalence of osteoporosis in the elderly over 65 years of age was $32 \%$, with $10.7 \%$ in men and $51.6 \%$ in women [6]. Patients with osteoporosis usually present with fractures after low-energy 
trauma, such as a fall on the ground level, commonly of the distal radius, which may precede more severe fractures, such as fractures of the neck of femur [7]. Nevertheless, osteoporotic patients are at risk of premature death [8]. Therefore, osteoporotic fractures have become a worldwide problem and have dramatically increased the burden on limited healthcare resources. Great efforts have been made to develop better treatments for osteoporotic fractures.

Preventing the first fragility fracture is the most cost-effective strategy for addressing osteoporosis [9]. For this purpose, determining the risk factors for osteoporotic fractures is important to identify individuals with a high risk of fractures.

Currently, the Fracture Risk Assessment Tool (FRAX), a computer-based algorithm that uses selected clinical risk factors to estimate a 10-year probability of hip and major osteoporotic fracture, is the most commonly used clinical tool recommended by the World Health Organization (WHO) for clinical decision making [10]. BMD combined with clinical risk scores can predict individuals who are at a high risk of fragility fracture [2]. The FRAX tool has been made available for 66 countries [11]. However, it is still necessary for each country to develop its own version of FRAX considering the differences in race and socioeconomic situations [12]. Thus far, there has been no specific Chinese recommendation for FRAX intervention thresholds based on updated Chinese epidemiological data on fracture incidence and its influencing factors [13]. In particular, some risk factors included in FRAX may be specific to Caucasians and may not be applicable to Asians [14].

In this study, the risk factors of primary osteoporotic fractures were examined using a case-control design. In addition to the parameters required by FRAX, other potential risk factors, including exercise habits, smoking status, diabetes history, pharmacohistory, muscle strength, and age-adjusted Charlson comorbidity index (aCCI), were also collected through a questionnaire and compared. The objectives of this study were to (1) identify the major risk factors for primary fracture and to (2) evaluate the positive predictive value of FRAX.

\section{Methods}

\subsection{Subjects}

This clinical study was reviewed and approved by the ethical committee for medical research of the University of Hong Kong-Shenzhen Hospital and registered at the Chinese Clinical Trail Registry (Clinical registration number: ChiCTR2000030927). A case-control study (with a level of evidence of 3) was conducted in which each subject underwent BMD measurement and questionnaire evaluation at the University of Hong Kong-Shenzhen Hospital. The patients were enrolled between January 2018 and April 2020 and assigned to either the fracture group or the control group according to predetermined criteria.

The fracture group inclusion criteria were as follows: (1) first-time fracture, (2) fracture occurring within one month, and (3) age 50-85 years at the time of enrollment. The fracture group exclusion criteria were as follows: (1) pathological fractures; (2) high-energy traumatic fracture; (3) secondary osteoporotic fractures, such as those caused by endocrine and metabolic diseases, connective tissue diseases, kidney diseases, digestive tract diseases, neuromuscular system diseases, blood system diseases, organ transplantation, and drugs; (4) prisoners; (5) mental disorders; and (6) cognitively unable to understand the study.

The control group inclusion criteria were (1) age 50-85 years old at the time of enrollment and (2) no history of fracture. The control group exclusion criteria were (1) prisoners, (2) mental disorders, and (3) poor cognitive ability.

\subsection{Data Source}

Dual-energy X-ray absorptiometry (DEXA) and all forms of questionnaire evaluations, which consisted of basic information, drug history, chronic history, fall risk assessment and fracture site, age-adjusted Charlson comorbidity index (aCCI), modified Barthel Index (MBI), and a locally validated 3-level version of EuroQol five dimensions questionnaire (EQ-5D-3L, which was converted to time-trade-off (TTO) in data analysis.), were used for the patients' assessment.

\subsection{Data Collection and Analysis}

In this study, osteoporosis was determined using DEXA with a BMD T-score less than or equal to -2.5 , and osteopenia was defined as a T-score between -1.0 and -2.5 , as recommended by the WHO. Subjects with a T-score greater than -1.0 were considered normal $[15,16]$. Pre-injury MBI and EQ-5D-3L were measured using questionnaires.

Statistical analyses were performed using SPSS. Continuous variables were expressed as the mean \pm standard deviation or median (range). Categorical variables were compared using Pearson's chi-square test, and continuous variables were compared using an independent T-test in the univariate analysis. The significant risk factors were further evaluated using multivariate logistic regression. The covariates that were controlled during multivariate analysis for outcome analysis were age, smoking, alcohol intake, parents' previous fracture history, diabetes mellitus type II, vitamin D or calcium intake, aCCI, muscle strength test, MBI, EQ-5D-3L, and DEXA. Statistical significance was set to $\mathrm{p}<0.05$.

\section{Results}

\subsection{Patient Characteristics}

The clinical characteristics of the patients recruited in the fracture and control groups are summarized in Supplementary Table 1. A total of 84 subjects, 41 in the fracture group and 43 in the control group, were recruited. Among the subjects, 19 were men and 65 were women, with a median age of 63.5 years (range 51-85 years). Table 1 presents the details of their baseline characteristics.

Osteoporosis was defined as a BMD T-score lower than 2.5 in at least one region of measurement [17]. The numbers of osteoporotic, osteopenia, and normal patients were 47, 28 , and 9 , respectively. 
The demographic statuses of the two groups were compared and are shown in Table 1. According to the univariate analysis, the fracture group had greater age $(p<0.001)$, aCCI $(p=0.001)$, muscle strength $(\mathrm{p}<0.001)$, TTO $(p=0.001)$, and BMD $(p=0.001)$; a higher incidence of smoking status $(p=0.045)$, alcohol intake $(p=0.013)$, parental fracture history $(p=0.031)$, diabetes mellitus type II $(p=0.036)$, vitamin D intake $(p=0.045)$, and calcium intake $(p=0.001)$; and a subnormal Barthel Index $(p=0.009)$.

Table 2 shows the fracture types in the fracture group. Femoral neck fractures, intertrochanteric fractures, thoracolumbar fractures, and distal radius fractures were the main fracture forms in the fracture group.

Table 1. Clinical characteristics.

\begin{tabular}{|c|c|c|c|c|}
\hline Parameters & Total numbers for two groups & Fracture group & Control group & $P$ \\
\hline Frequency & 84 & 41 & 43 & \\
\hline Gender & & & & 0.088 \\
\hline Male & $19(22.62 \%)$ & $6(14.63 \%)$ & $13(30.23 \%)$ & \\
\hline Female & $65(77.38 \%)$ & $35(85.37 \%)$ & $30(69.77 \%)$ & \\
\hline Age, years, median (range) & $63.5(51-85)$ & $71(52-85)$ & $61(51-74)$ & $0.000 * * *$ \\
\hline Body mass index $\left(\mathrm{kg} / \mathrm{m}^{2}\right)$ & $23.59 \pm 2.82$ & $23.31 \pm 2.71$ & $23.87 \pm 2.93$ & 0.365 \\
\hline Smoking & & & & $0.045^{*}$ \\
\hline No & $80(95.24 \%)$ & $41(100 \%)$ & $39(90.7 \%)$ & \\
\hline Yes & $4(4.76 \%)$ & $0(0)$ & $4(9.3 \%)$ & \\
\hline Alcohol intake & & & & $0.013^{*}$ \\
\hline No & $78(92.86 \%)$ & $41(100 \%)$ & $37(86.05 \%)$ & \\
\hline Yes & $6(7.14 \%)$ & $0(0)$ & $6(13.95 \%)$ & \\
\hline Parents Previous Fracture History & & & & $0.031^{*}$ \\
\hline No & $76(90.48 \%)$ & $40(97.56 \%)$ & $36(83.72 \%)$ & \\
\hline Yes & $8(9.52 \%)$ & $1(2.44 \%)$ & $7(16.28 \%)$ & \\
\hline Amount of exercise & & & & 0.0897 \\
\hline None & $25(29.76 \%)$ & $13(31.71 \%)$ & $12(27.91 \%)$ & \\
\hline Less & $39(46.43 \%)$ & $19(46.34 \%)$ & $20(46.51 \%)$ & \\
\hline More & $20(23.81 \%)$ & $9(21.95 \%)$ & $11(25.58 \%)$ & \\
\hline Diabetes mellitus type II & & & & $0.036^{*}$ \\
\hline No & $69(82.14)$ & $30(73.17 \%)$ & $39(90.7 \%)$ & \\
\hline Yes & $15(17.86)$ & $11(26.83 \%)$ & $4(9.3 \%)$ & \\
\hline Rheumatoid arthritis & & & & 0.283 \\
\hline No & $80(95.24 \%)$ & $38(92.68 \%)$ & $42(97.67 \%)$ & \\
\hline Yes & $4(4.76 \%)$ & $3(7.32 \%)$ & $1(2.33 \%)$ & \\
\hline $\mathrm{aCCI}$ & & & & $0.001 * *$ \\
\hline $0 \sim 1$ & $24(28.57 \%)$ & $7(17.08 \%)$ & $17(39.53 \%)$ & \\
\hline $2 \sim 3$ & $40(47.62 \%)$ & $17(41.46 \%)$ & $23(53.49 \%)$ & \\
\hline$>=4$ & $20(23.81 \%)$ & $17(41.46 \%)$ & $3(6.98 \%)$ & \\
\hline Vitamin D intake & & & & $0.045^{*}$ \\
\hline No & $80(95.24 \%)$ & $41(100 \%)$ & $39(90.7 \%)$ & \\
\hline Yes & $4(4.76 \%)$ & $0(0)$ & $4(9.3 \%)$ & \\
\hline Calcium intake & & & & $0.001 * *$ \\
\hline No & $74(88.1 \%)$ & $41(100 \%)$ & $33(76.74 \%)$ & \\
\hline Yes & $10(11.9 \%)$ & $0(0)$ & $10(23.26 \%)$ & \\
\hline Drug for Hypertension & & & & 0.703 \\
\hline No & $59(70.24 \%)$ & $28(68.29 \%)$ & $31(72.09 \%)$ & \\
\hline Yes & $25(20.76 \%)$ & $13(31.71 \%)$ & $12(27.91 \%)$ & \\
\hline Drugs for diabetes & & & & 0.109 \\
\hline No & $71(84.52 \%)$ & $32(78.05 \%)$ & $39(90.7 \%)$ & \\
\hline Yes & $13(15.48 \%)$ & $9(21.95 \%)$ & $4(9.3 \%)$ & \\
\hline Muscle strength test* & & & & $0.000 * * *$ \\
\hline Unqualified $(<10)$ & $28(33.33 \%)$ & $21(51.22 \%)$ & $7(16.28 \%)$ & \\
\hline Qualified (10-20) & $13(15.48 \%)$ & $9(21.95 \%)$ & $4(9.3 \%)$ & \\
\hline Excellent $(>=20)$ & $43(51.19 \%)$ & $11(26.83 \%)$ & $32(41.42 \%)$ & \\
\hline MBI & & & & $0.009^{* *}$ \\
\hline 61 99 & $6(7.14 \%)$ & $6(14.63 \%)$ & $0(0 \%)$ & \\
\hline 100 (Normal) & $78(92.86 \%)$ & $35(85.37 \%)$ & $43(100 \%)$ & \\
\hline TTO, Mean \pm SD & $0.61 \pm 0.06$ & $0.63 \pm 0.05$ & $0.59 \pm 0.07$ & $0.001 * *$ \\
\hline DEXA, T & & $-3.24 \pm 1.07$ & $-2.29 \pm 1.05$ & $0.001 * *$ \\
\hline Osteoporosis $(\mathrm{T} \leq-2.5)$ & $47(55.95 \%)$ & $33(80.49 \%)$ & $14(32.56 \%)$ & \\
\hline Osteopenia $(-2.5<\mathrm{T} \leq-1)$ & $28(33.33 \%)$ & $7(17.07) \%$ & $21(48.84 \%)$ & \\
\hline $\operatorname{Normal}(\mathrm{T}>-1)$ & $9(10.72 \%)$ & $1(2.44 \%)$ & $8(18.6 \%)$ & \\
\hline
\end{tabular}

Muscle strength test*: Unqualified means supine leg lift less than 10 times per minute; Qualified means 10-20 times per minute; Excellent means more than 20 times per minute. 
Table 2. Fracture types in the fracture group.

\begin{tabular}{ll}
\hline Fracture types & Fracture group $(\mathbf{n}=\mathbf{4 1})$ \\
\hline Femoral neck fracture & $9(21.95 \%)$ \\
Intertrochanteric fracture & $6(14.63 \%)$ \\
Compression fracture of lumbar vertebra & $8(19.51 \%)$ \\
Compression fracture of thoracic vertebra & $6(14.63 \%)$ \\
Thoracolumbar vertebral compression & $4(9.77 \%)$ \\
fractures & $6(14.63 \%)$ \\
Distal Radius Fractures & $1(2.44 \%)$ \\
Tibial proximal fracture & $1(2.44 \%)$ \\
Proximal humeral fractures & \\
\hline
\end{tabular}

\subsection{Data Analysis}

Table 3 shows the binary logistic regression analysis of the factors related to osteoporosis fracture. The factors included in the model were age, smoking, alcohol intake, parents' previous history, diabetes II, vitamin D or calcium intake, aCCI, muscle strength test, MBI, TTO, and DEXA.

Patients who were diagnosed with osteoporosis $(\mathrm{T} \leq-2.5)$ showed a greater risk of fracture $(\mathrm{OR}=15.96,95 \% \mathrm{CI}=0.33$ 776.48, $P=0.001$ ) (Figure 1). The binary logistic regression analysis showed that DEXA-diagnosed osteoporosis $(\mathrm{T} \leq-2.5)$ was the independent fracture risk factor. The fracture probability of a low $\mathrm{T}$ value $(\mathrm{T} \leq-2.5)$ was high.

Table 3. Binary logistic regression analysis of the factors related to osteoporosis fracture.

\begin{tabular}{lll}
\hline Parameters & OR $(\mathbf{9 5 \%} \% \mathbf{C I})$ & $\boldsymbol{P}$ \\
\hline DEXA, T & & $0.001^{* *}$ \\
Normal $(\mathrm{T}>-1)$ & 1.00 & \\
Osteoporosis $(\mathrm{T} \leq-2.5)$ & $15.96(0.33-776.48)$ & \\
\hline
\end{tabular}

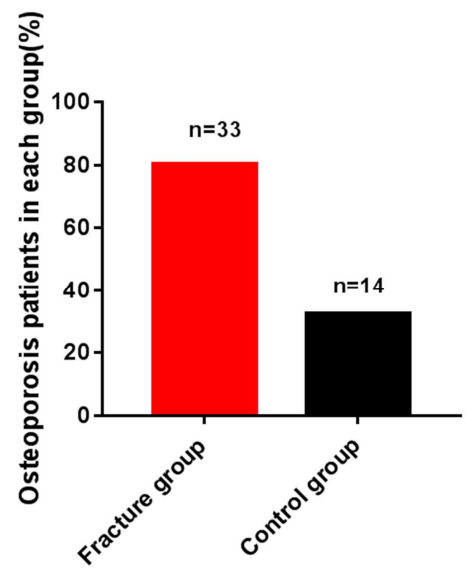

Figure 1. Ratio of osteoporosis patients in each group.

Table 4. Covariance analysis of factors related to DEXA.

\begin{tabular}{lll}
\hline Parameters & F & $\boldsymbol{P}$ \\
\hline Age*MBI*TTO & 0.616 & 0.607 \\
Age & 3.57 & 0.033 \\
Muscle strength*MBI*TTO & 0.361 & 0.781 \\
Muscle strength & 5.884 & 0.004 \\
aCCI*MBI*TTO & 0.282 & 0.838 \\
aCCI & 3.391 & 0.039 \\
\hline
\end{tabular}

Table 4 shows the covariance analysis of the factors related to DEXA. The effects of age, muscle strength, and Charlson index on DEXA were statistically significant.

Figures 2-4 show the effects of age, muscle strength, and Charlson index on DEXA. Significant differences were found between the 70-85 age group and the 60-69 and 50-59 age groups but not between the 60-69 and 50-59 age groups (Figure 2). Significant differences were found between the excellent muscle strength group and the qualified muscle strength and the unqualified muscle strength groups but not between the qualified muscle strength group and the unqualified muscle strength group (Figure 3). A significant difference was found between aCCI scores $\geq 4$ and $0-1$ group but not between aCCI scores 2-3 and $\geq$ 4 and the $0-1$ group (Figure 4 ).

Based on the above analysis, we can come to conclusion that people old over 60 , muscle strength test supine leg lift less than 20 times per minute, aCCI scores greater than or equal to 2 , had lower DEXA T values $(\mathrm{T} \leq-2.5)$. So there people have a higher risk of fracture.

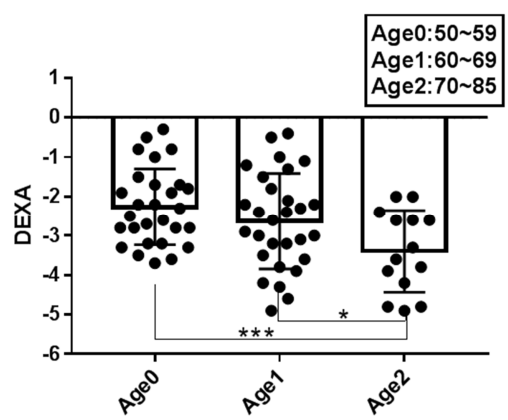

Figure 2. Effects of age on DEXA.

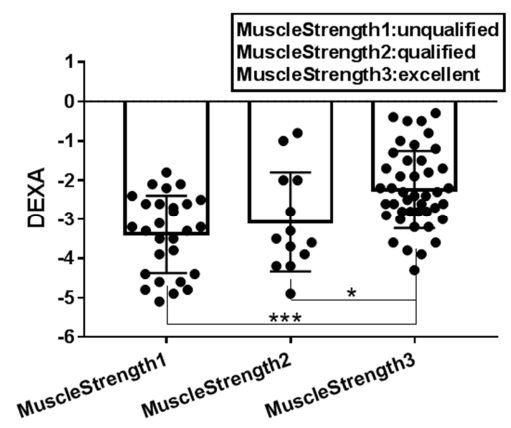

Figure 3. Effects of muscle strength on DEXA.

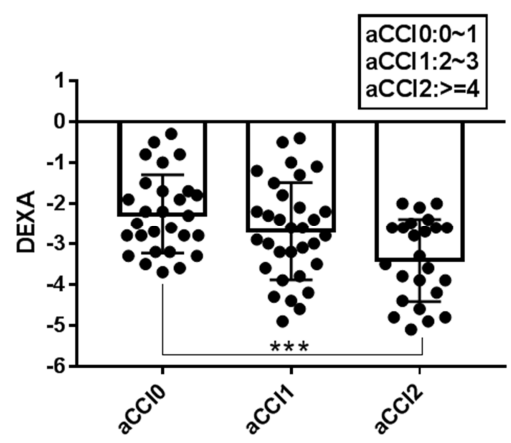

Figure 4. Effects of aCCI on DEXA.

The 10-year probability of a major osteoporotic fracture calculated by FRAX is shown in Figure 5. The positive 
predictive value of FRAX at the time of the patients' recruitment was $9.87 \%$. Significance between the two groups was observed for a major osteoporotic fracture. The incidences of major osteoporotic fractures in the fracture group were significantly higher than those in the control group.

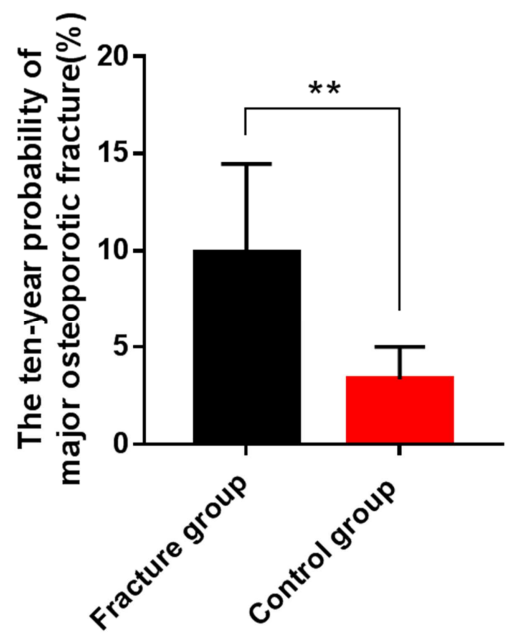

Figure 5. The ten-year probability of major osteoporotic fracture in two groups.

\section{Discussion}

A proper intervention can reduce fracture risk. To prevent fractures, a common clinical practice is to recommend that people have both $30 \mathrm{~min}$ of walking exercise and gentle anaerobic exercises per day. Patients should also be counseled to avoid cigarette smoking and excessive alcohol intake. The recommended dietary allowance (RDA) for calcium is 1,000 , 1,000 , and $1,200 \mathrm{mg} / \mathrm{d}$ for women above 50 years old, for men aged 19-70 years old, and for men above 70 years old. The RDA for vitamin D is $600 \mathrm{IU} / \mathrm{d}$ for men and women aged 19 70 years and $800 \mathrm{IU} / \mathrm{d}$ for those older than 70 years [1]. In some cases, aside from recommending daily exercises and taking calcium and vitamin $\mathrm{D}$, doctors also use drugs to either inhibit osteoclasts or promote osteoblasts based on patients' osteogenic and osteoclastic activity.

No matter what kind of intervention is applied, the most important thing is to identify subjects who are at high risk of osteoporosis fractures. The FRAX tool has been used globally for the purpose of fracture risk assessment [16], in which risk factors, including age, sex, height, weight, prior fracture, parental fracture history, smoking, drinking, taking adrenocortical hormone, rheumatoid arthritis, secondary osteoporosis, and BMD, are integrated to provide a 10-year probability of hip fracture and major osteoporotic fracture. Although the FRAX algorithm does not consider recency, number, severity, or site of prior fracture, it is still helpful in identifying individuals with a high risk of bone fracture as an indicator for clinical treatment [16]. Furthermore, country-specific FRAX models evaluate and revise the algorithm according to performance in different regions, making FRAX the most prevalent prognostication tool so far [1].

However, the accuracy of FRAX still needs to be improved. Lelia et al. used the Australian FRAX scores to evaluate women with impaired fasting glucose and diabetes and found that FRAX calculations, including BMD, might not be accurate in estimating fractures in those with diabetes [18]. Povoroznyuk et al. found that the Ukrainian FRAX model could enhance the accuracy of determining fracture probability among the Ukrainian population and help guide decisions about treatment [19]. Moreover, it is unclear whether FRAX is useful in making individualized treatment decisions, although FRAX has been recalibrated in many Asian regions using the corresponding population-based data in these regions [14]. Furthermore, the epidemiological survey data of osteoporosis fractures are insufficient and incomplete, and fracture incidence is underestimated. To better adapt to the health of the Chinese population, other potential risk factors were also evaluated in this study, such as drug history, chronic history, fall risk assessment, fracture site, aCCI, MBI, and EQ-5D-3L.

Our findings showed that risk factors, including age, smoking status, alcohol intake, family history of osteoporotic fracture, diabetes mellitus type II, aCCI, vitamin D intake, calcium intake, muscle strength, MBI, EQ-5D-3L, and BMD, had a significant difference between the fracture and control groups. Moreover, DEXA-diagnosed osteoporosis $(\mathrm{T} \leq-2.5)$ was the independent fracture risk factor. The effects of age, muscle strength, and Charlson index on DEXA were statistically significant. People old over 60, muscle strength test supine leg lift less than 20 times per minute, aCCI scores greater than or equal to 2 , had lower DEXA $T$ values $(T \leq-2.5)$.

The possibility of major osteoporotic fractures as predicted by FRAX was greater in the fracture group than in the control group. However, the positive predictive value of FRAX for a major osteoporotic fracture was only $9.87 \%$, suggesting that FRAX underestimated fracture risks. Therefore, a more effective prediction tool or an improved FRAX should be developed.

\section{Conclusion}

Our results showed that DEXA-diagnosed osteoporosis (T $\leq-2.5$ ) was the independent fracture risk factor. People old over 60 , muscle strength test supine leg lift less than 20 times per minute, aCCI scores greater than or equal to 2 , had lower DEXA $T$ values $(T \leq-2.5)$. This study suggested that FRAX underestimated fracture risks and FRAX should be improved further in combination with other risk factors, including aCCI, calcium intake, and muscle strength.

\section{Limitations and Suggestions}

Current evidence suggests that a decision aid incorporating FRAX may improve patient knowledge, but no effect on the rates of treatment initiation or adherence has been demonstrated [20]. This work was a single-center study, showed social economic status bias, and featured a limited number of cases. Therefore, multi-center studies are necessary in the future. A large Chinese database of osteoporosis should be established by standardizing the data format among 
hospitals in mainland China. By combining artificial intelligence and big data, it is possible to reveal more key risk factors for osteoporotic bone fractures and to help improve clinical treatments for patients.

\section{Conflicts of Interest}

All authors declare that there is no conflict of interest.

\section{Acknowledgements}

This work was financially supported by Guangdong Basic and Applied Basic Research Foundation (2018A030313888), National Natural Science Foundation of China (81802959), Shenzhen Science and Technology Funding (JCYJ20170413162540673, JSGG20180507183242702 and JCYJ20200109150420892), HKU-SZH Fund for Shenzhen Key Medical Discipline (SZXK2020084), Sanming Project of Medicine in Shenzhen "Team of Excellence in Spinal Deformities and Spinal Degeneration" (SZSM201612055) and Guangdong High Level-Hospital Program (HKU-SZH201901021).

\section{References}

[1] Ensrud KE, Crandall CJ. Osteoporosis. Ann Intern Med. 2017; 167 (3): ITC17-ITC31. doi: 10.7326/AITC201708010.

[2] Fuggle NR, Curtis EM, Ward KA, Harvey NC, Dennison EM, Cooper C. Fracture prediction, imaging and screening in osteoporosis. Nat Rev Endocrinol. 2019; 15 (9): 535-547. doi: 10.1038/s41574-019-0220-8.

[3] Liu J, Curtis EM, Cooper C, Harvey NC. State of the art in osteoporosis risk assessment and treatment. $J$ Endocrinol Invest. Published online 2019. doi: 10.1007/s40618-019-01041-6.

[4] Feehan J, Al Saedi A, Duque G. Targeting fundamental aging mechanisms to treat osteoporosis. Expert Opin Ther Targets. 2019; 23 (12): 1031-1039. doi: $10.1080 / 14728222.2019 .1702973$.

[5] Aspray TJ, Hill TR. Osteoporosis and the ageing skeleton. Subcell Biochem. 2019; 91: 453-476. doi: 10.1007/978-981-13-3681-2_16.

[6] Chinese Society of Osteoporosis and Bone Mineral Research, Chinese Medical Association, Epidemiological survey of osteoporosis in China and release of the results of "healthy Skeleton, special action", Chin J Osteoporosis \& Bone Miner Res, 2019; 12 (4): 317-318.

[7] Niempoog S, Sukkarnkosol S, Boontanapibul K. Prevalence of osteoporosis in patients with distal radius fracture from low-energy trauma. Malaysian Orthop J. 2019; 13 (3): 15-20. doi: 10.5704/MOJ.1911.003.
[8] González-Quevedo D, Bautista-Enrique D, Pérez-del-Río V, Bravo-Bardají M, García-de-Quevedo D, Tamimi I. Fracture liaison service and mortality in elderly hip fracture patients: a prospective cohort study. Osteoporos Int. 2020; 31 (1): 77-84. doi: 10.1007/s00198-019-05153-w.

[9] Kerschan-Schindl K. Prävention und Rehabilitation der Osteoporose. Wiener Medizinische Wochenschrift. 2016; 166 (1-2): 22-27. doi: 10.1007/s10354-015-0417-y.

[10] Iconaru L, Moreau M, Kinnard V, et al. Does the Prediction Accuracy of Osteoporotic Fractures by BMD and Clinical Risk Factors Vary With Fracture Site? JBMR Plus. 2019; 3 (12): e10238. doi: $10.1002 / \mathrm{jbm} 4.10238$.

[11] Kanis JA, Harvey NC, Johansson H, et al. A decade of FRAX: how has it changed the management of osteoporosis? Aging Clin Exp Res. 2020; 32 (2): 187-196. doi: 10.1007/s40520-019-01432-y.

[12] Lekamwasam S. The diversity of Fracture Risk Assessment Tool (FRAX)-based intervention thresholds in Asia. Osteoporos Sarcopenia. 2019; 5 (4): 104-108. doi: 10.1016/j.afos.2019.12.002.

[13] Liu S, Chen R, Ding N, et al. Setting the new FRAX reference threshold without bone mineral density in Chinese postmenopausal women. $J$ Endocrinol Invest. 2020; (0123456789). doi: 10.1007/s40618-020-01315-4.

[14] Cheung C-L. FRAX-based intervention thresholds in Asia: Now and future. Osteoporos Sarcopenia. 2019; 5 (4): 103. doi: 10.1016/j.afos.2019.12.003.

[15] Aletaha D, Neogi T, Silman AJ, et al. 2010 Rheumatoid arthritis classification criteria: An American College of Rheumatology/European League Against Rheumatism collaborative initiative. Ann Rheum Dis. 2010; 69 (9): 1580-1588. doi: 10.1136/ard.2010.138461.

[16] Lai EL, Huang WN, Chen HH, et al. Ten-year fracture risk by FRAX and osteoporotic fractures in patients with systemic autoimmune diseases. Lupus. 2019; 28 (8): 945-953. doi: $10.1177 / 0961203319855122$.

[17] Kanis JA. An update on the diagnosis of osteoporosis. Curr Rheumatol Rep. 2000; 2 (1): 62-66. doi: 10.1007/s11926-996-0070-y.

[18] de Abreu LLF, Holloway-Kew KL, Sajjad MA, Kotowicz MA, Pasco JA. FRAX (Australia) scores in women with impaired fasting glucose and diabetes. Bone Reports. 2019; 11 (July): 100223. doi: 10.1016/j.bonr.2019.100223.

[19] Povoroznyuk V V., Grygorieva N V., Kanis JA, et al. Epidemiology of hip fracture and the development of FRAX in Ukraine. Arch Osteoporos. 2017; 12 (1). doi: 10.1007/s11657-017-0343-2.

[20] Montori VM, Shah ND, Pencille LJ, et al. Use of a decision aid to improve treatment decisions in osteoporosis: The osteoporosis choice randomized trial. Am J Med. Published online 2011. doi: 10.1016/j.amjmed.2011.01.013. 